\title{
Fault Diagnosis of Steam Turbine Vibration Based on Fault Tree Analysis
}

\author{
Xiaodong Zhu ${ }^{1, a}$, Xiu Tan ${ }^{2, b}$, Wei Jiang ${ }^{3, c}$ and Yuanyue Bu ${ }^{4, d}$ \\ 1,2Hudian Electric Power Research Institute, Hangzhou, China \\ ${ }^{3,4}$ Inner Mongolia Daihai Electric Power Generation Co. Ltd., Liangcheng , China

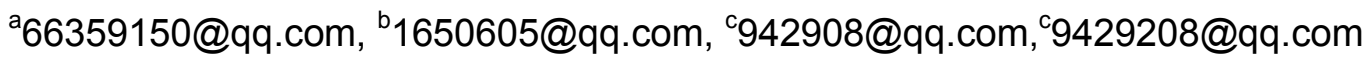

\section{Keywords: Fault tree; Steam turbine vibration; Fault diagnosis; Research}

\begin{abstract}
In view of the important status of steam turbine in power system, continue to strengthen the theoretical research of the technology of fault diagnosis, to avoid accidents so as to improve the operation reliability of steam turbine, it is necessary, and combined with the needs of fault diagnosis, the fault tree describes the logical relationship between the fault, more vivid, simple and clear. This paper studies the application of the technology of fault diagnosis and fault tree analysis of the technology in recent years, analyzed the vibration fault of turbine generator, based on this, the fault tree is applied to the field of vibration fault diagnosis of steam turbine, and the turbine rotor misalignment fault tree qualitative analysis as an example, calculate the minimum cut set. It is proved that it is feasible to apply the fault tree to the vibration fault diagnosis of steam turbine.
\end{abstract}

\section{Introduction}

With the development of economy, the demand for electric power is in the growth of large steam turbine group is born, the increasing unit capacity to present its basic trend of slowing, but the research on the method of fault diagnosis is still in development, especially the diversification of the turbine vibration faults, which requires us to summarize and study the existing fault a new fault mechanism and diagnosis method. The forced vibration in the operation of the steam turbine generator unit usually includes resonance, mechanical loosening, electromagnetic excitation, rotor imbalance, and the center of the shaft is not correct. In the case of forced vibration, the vibration frequency is the same as that of the external exciting force. The self-excited vibration, from the literal meaning, is a kind of vibration and is irrelevant to the outside of the rotating machinery, but there is a real and a vibration has nothing to do with the external force, under normal circumstances is not going to happen, it is essentially belongs to abnormal vibration, must take the necessary measures to control and eliminate. The cause of the self-excited vibration of rotating machinery to the rotor support system has a feedback loop to unload. The feedback link causes the rotor to obtain energy from the rotation, and converts the vibration energy to a certain frequency to maintain the vibration. The frequency of the self-excited vibration is completely independent of the frequency of the external force, and is consistent with the natural frequency of the mechanical system. The usual vibration includes the self-excited vibration of the bearing, the steam excitation and the friction whirl.

\section{Vibration Fault Diagnosis of Steam Turbine}

Under normal circumstances, as long as the abnormal state of the unit, will inevitably be reflected in the level of change or abnormal vibration, which is evident in the abnormal vibration frequency. In fact, the components of each vibration spectrum and its change are all indicative of the failure. Vibration fault diagnosis is based on the vibration characteristics and vibration characteristics of the analysis of the causes of abnormal vibration prediction and inference. However, in order to correctly diagnose the cause of vibration, in addition to the use of advanced means for the analysis of vibration signals, the knowledge about the mechanism of abnormal vibration and the rich field experience is also indispensable. In general, we first need to identify the characteristic matrix of the 
vibration fault, because we can see the flat rate characteristics and vibration characteristics of the vibration fault. And it is an important objective basis for off-line and on-line vibration fault diagnosis. Again, we need to sum up the general diagnostic methods of vibration faults. There are two main categories: numerical method and knowledge-based method. The numerical methods include probability statistics, pattern recognition, organization theory, neural network and fuzzy mathematics, which cannot do without computer control, and these methods combined with computer after the formation of self diagnosis system, this system does not need to be related to deep-seated fault inception mechanism and fault knowledge, only one a learning cycle of the fault data, then the diagnosis model, calculator symptom vector, the vector of reason. As a result of diagnosis, we generally think that the larger the result of the cause vector, the greater the probability of the cause of the fault, or the high confidence of the diagnostic results. In general, we can only use the method based on knowledge to understand and apply the fault mode, corrective behavior pattern and case description. The failure mode of the general symptoms including fault and causality tree method.

\section{Vibration Fault Diagnosis of Steam Turbine Unit}

The key to solve the vibration problem is to understand the origin of vibration. The historical experience and lessons of unit vibration diagnosis and handling instructions, the qualitative vibration fault is generally not difficult, but the specific reasons to determine the fault is due to the influence of the unit structure by insight and understanding and experience of the staff and other factors, is a miscarriage of justice has occurred, and can not take correct treatment of vibration the. In some cases, there are some differences between the vibration characteristics reflected by the fault and the characteristics of the theoretical research. Therefore, in the diagnosis and treatment work of vibration, vibration analysis of the personnel of the need to master certain basic vibration and rotor dynamics theory and scientific analysis ability, but also have rich experience in the field, familiar with the structure and vibration characteristics of the unit, so that it can express the exact cause of this high failure, put forward right radical measures. In order to master the vibration fault diagnosis technology of the unit, and to be competent in the field of vibration diagnosis and processing, some basic principles can be used as the basis for the vibration diagnosis of the unit.

Familiar with Vibration Testing Instrument. Vibration measurement data is the basis of vibration analysis. The vibration analyst should be familiar with all kinds of off line and on line instruments used in the field, and can skillfully use the vibration test of the Xi'an instrument to carry out the test of the shaft vibration. In addition, still should be able to from the current widespread installation of large unit vibration transient data management system (TDM) in out real-time and historical data and related analysis of vibration characteristic curve, comparison analysis and reference.

Understand the Basic Mechanical Characteristics. The vibration analysis of the personnel of the unit should be familiar with the working principle, its structure layout and structural design parameters (including bearing clearance, seal gap, etc.) with steady understanding unit (normal with full load and part load) and mechanical characteristics in transient operation. Including the critical speed of the shaft and the shaft type, the amount of damping in the system, whether the unit is unstable, the sensitivity of each part of the imbalance response and the weight of the lag angle, the relationship between vibration and load, as well as the vibration limit value. The mechanical characteristics of the steam turbine generator set are generally obtained from the calculation of the vibration characteristics of the shafting and the field measurement and analysis. Factory to obtain the mechanical properties in the design process, he must meet the following three basic requirements: critical speed can not be close to the working speed; in the whole speed range of the amplitude of vibration should be lower, to ensure the necessary clearance, prevent rubbing, and the rotor to force smaller rotor and bearing; the bearing system should be able to suppress the self-excited vibration (oil film oscillation and steam turbinedynamic etc.). The mechanical characteristics of the steam turbine are determined by the characteristics of the rotor, bearing and foundation. Therefore, some possible vibration fault and rotor, bearings and other parts of the 
architecture, compares the characteristics of the vibration characteristics and manufacturing plant occupied by measuring field calculation, can not only find the fault reason, but also conducive to completely solve the problem, especially in the design must change the components should be used when the original calculating method the effect of the improved estimation.

Familiar with the Vibration Response and Vibration Characteristics of the Typical Fault. Generally, the vibration characteristic curve is determined by the design of the rotating machinery itself, the installation condition of the structural material and the river. Once these factors are changing in the operation, the vibration characteristics will change accordingly. Generally, the vibration characteristics can be solved by theoretical calculation, but empirical data are often used in practical applications. For each specific vibration fault, the vibration characteristics can be expressed in a certain extent, which is reflected in the vibration frequency spectrum, the axis orbit and the time domain waveform. The relationship between vibration and vibration characteristics of typical have obtained the experimental and statistical probability, the main vibration spectrum, including vibration fault icon corresponding with the maximum amplitude of vibration causes the direction and position of the table, and with the rise of unit, vibration amplitude table speed reduction process. Of course, the vibration characteristics of some vibration faults may be the same or similar, but can be further distinguished by analyzing the relationship between the vibration phase and the vibration and other non-vibration quantities.

Understand the Historical Events that Cause the Unit to Change. In addition to the sudden increase of the unit vibration caused by the failure of the turbine blades, there is a gradual development process. During this period of historical events, such as the turbine inlet, cold steam and other faults, as well as the maintenance of the unit, may have an impact on the vibration of the unit. Therefore, according to the change trend of the vibration, the query has a decisive influence on the vibration of the historical events, help to analyze the causes of vibration, to take appropriate measures to deal with.

Detection can Represent the Key Parameters of the Unit Change. Turbogenerator vibration often involves many factors, it should be the parameters and other operating parameters related to sugarcane farmers try, to find the vibration causes. For this reason, the vibration test and the external characteristic test are carried out to determine the key parameters which lead to the increase of vibration.

Process Data to Make it Easy to Understand. Vibration measurements can be obtained from the vibration data. In order to facilitate the use and vibration analysis, it is usually necessary to carry out mathematical treatment of these data. List the processed data in plain text and simple data table or graphic form, and experienced the typical medical vibration fault and vibration characteristics of relational tables in comparison, so we can easily determine the specific cause of the fault.

Reference to the Previous Unit Vibration and Handling Cases. Refer to the vibration diagnosis and treatment unit existing cases, can understand the fault types more, and learn from others with the diagnosis methods and reduce the vibration, vibration fault features to enhance the understanding of knowledge, experience and lessons learned, and the vibration diagnosis and treatment work after this help.

\section{Turbine Vibration Fault Tree Qualitative Analysis}

In the existing literature, qualitative analysis of the steam turbine vibration fault tree is more important, because we focus on the research result of bottom events of top event combinations, and find all the patterns caused by the top event, we can judge the hidden fault, so as to improve our design process of steam turbine, can guide us to carry out fault diagnosis in actual production, improve the turbine operation and maintenance program. And the result of the qualitative analysis is also helpful to the quantitative calculation, because qualitative analysis can also be used to find the minimum cut set of the system, in the quantitative analysis of the calculation, you can delete useless cut sets, the computation is greatly simplified calculation. It is obvious that the fault tree of the main vibration fault of the steam turbine is very simple in logic. This leads to the results of this article will be described in the following. 
Cut set and Minimum Cut Set. We know that the concept of set in mathematics, in the same way as the set of faults in a tree, is similar to that of a set of faults, because the cut set in a fault tree is the combination of the underlying events that cause the top event to occur in the common fault tree. The minimum cut set is a combination of a reasonable simplification of the bottom event, which is a kind of failure mode (caused by the occurrence of the common fault tree top event). In general, the common fault tree has only one minimal cut set. The minimum cut set of an event, which indicates that the event is a one to one relationship with the top event. If the minimum cut set has two or more components, it is necessary that these events occur at the same time, the top event will occur in the two.

Calculation Method of Minimum Cut Set. In this paper we use the qualitative analysis method of fault tree down, according to the basic theory of descending method, fault tree, the number of cut sets to see or increase a few days, and the cut set capacity is related to the number of gate, which cut capacity is the number of bottom events. According to the related literature, we summarize the method of descending steps: the first step is to start from the top event of fault tree, from the top to the bottom of the upper level event replacement for the next level event; second step parallel write all input events encountered and, at the same time in the process to write in tandem or input events, until all logic gates are changed to the bottom event, the last column represents all cut sets, the minimum cut set by all cut sets and simplified absorption. As shown in Fig. 1, the fault tree of the rotor of the turbine is not used, and the cut set, the minimum cut set.

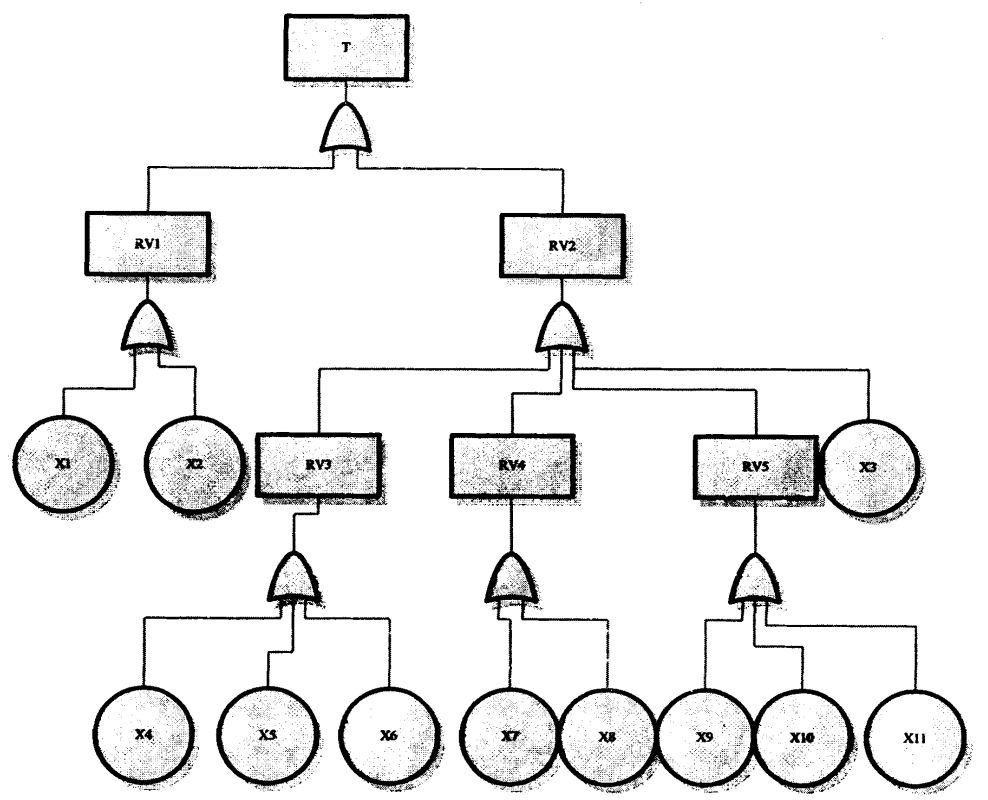

Figure 1. fault tree of turbine rotor misalignment

The meaning of the symbols in the fault tree diagram is shown in Table 1.

Table 1 Symbol meaning of rotor misalignment fault tree

\begin{tabular}{|l|l|l|l|}
\hline Symbol & Meaning & Symbol & Meaning \\
\hline T & Turbine rotor misalignment & X4 & Uneven base heating \\
\hline RV1 & Cold misalignment & X5 & Rotor weight distribution is not uniform \\
\hline RV2 & Thermal misalignment & X6 & Uneven foundation settlement \\
\hline RV3 & Uneven mass distribution & X7 & $\begin{array}{l}\text { Thermal expansion of each component } \\
\text { Twist }\end{array}$ \\
\hline RV4 & deformation & X8 & Frictional force on sliding surface \\
\hline RV5 & Tilt and side of bearing seat & X9 & during thermal expansion \\
\hline X1 & Machining error & X10 & Guide key wear \\
\hline X2 & Installation error & X11 & Condenser vacuum change \\
\hline
\end{tabular}


According to the descending method shows that obviously above only a logic gate, or gate. It is obviously that the use of the fault tree descending method to get the minimum cut set and cut set too simple, unbalanced rotor and Turbine rubbing fault tree minimal cut sets are the combination of all of its bottom events in order to illustrate the method, more specifically, at the same time we the unbalance fault tree to make some changes to the rotor rotor imbalance fault tree as an example, this will be a qualitative fault tree consists entirely of letters chart showing the process as shown in fig. 2 .

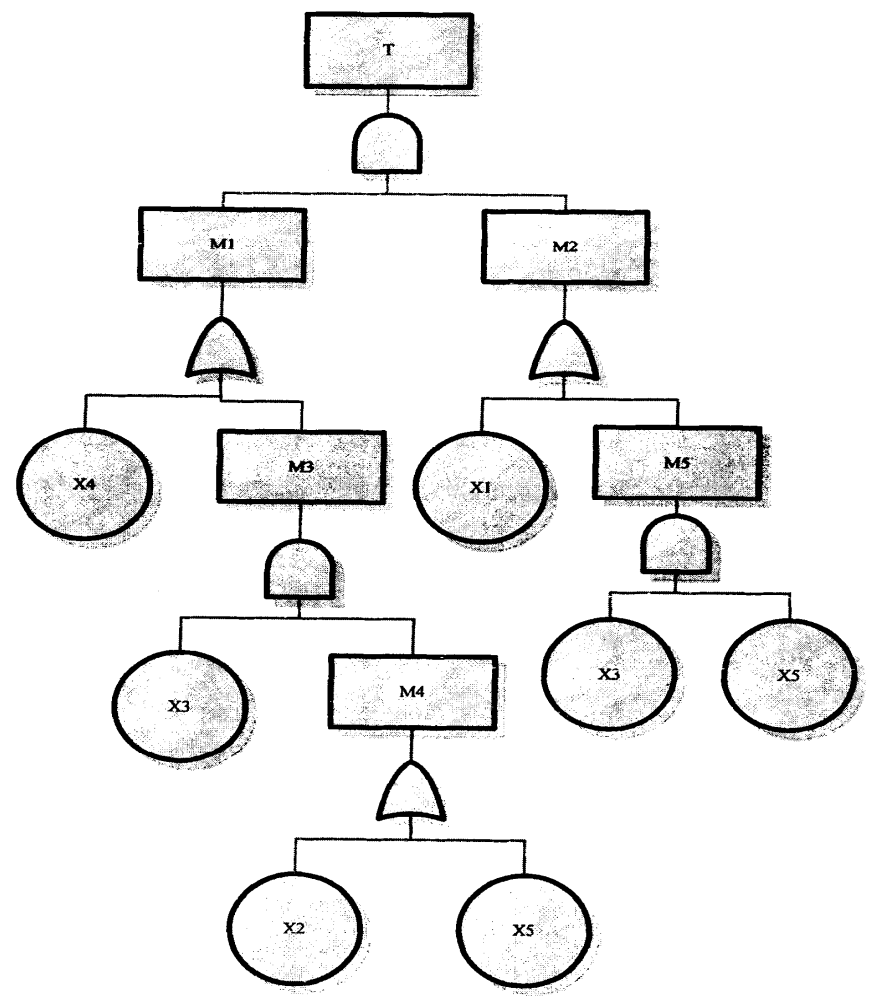

Fig. 2 fault tree diagram

The fault tree has six cut sets, $\{\mathrm{X} 4, \mathrm{X} 1\},\{\mathrm{X} 4, \mathrm{X} 3, \mathrm{X} 5\},\{\mathrm{X} 3, \mathrm{X} 2, \mathrm{X} 1\},\{\mathrm{X} 3, \mathrm{X} 5, \mathrm{X} 1\},\{\mathrm{X} 2, \mathrm{X} 3, \mathrm{X} 5\}$, $\{\mathrm{X} 3, \mathrm{X} 5\}, \quad$ simplification $\quad$ is $\mathrm{X} 2 \mathrm{X} 3 \mathrm{X} 5=\mathrm{X} 3 \mathrm{X} 2 \mathrm{X} 3 \mathrm{X} 5, \mathrm{X} 3 \mathrm{X} 5=\mathrm{X} 3 \mathrm{X} 5 \mathrm{X} 3 \mathrm{X} 5$, absorb $\mathrm{X} 3 \mathrm{X} 5+\mathrm{X} 4 \mathrm{X} 3 \mathrm{X} 5+\mathrm{X} 3 \mathrm{X} 5 \mathrm{XI}+\mathrm{X} 2 \mathrm{X} 3 \mathrm{X} 5=\mathrm{X} 3 \mathrm{X} 5$. The minimum cut set after simplification is $\{\mathrm{X} 3, \mathrm{X} 2, \mathrm{X} 1\},\{\mathrm{X} 3, \mathrm{X} 5\},\{\mathrm{X} 4, \mathrm{X} 1\}$. The minimum cut set is shown in Table 2.

Table 2 solving steps of minimum cut set

\begin{tabular}{|l|l|l|l|l|l|l|l|l|}
\hline \multicolumn{7}{|c|}{ Analysis steps } \\
\hline 1 & 2 & 3 & 4 & 5 & 6 & 7 & 8 & 9 \\
\hline T & M1M2 & X4M2 & X4X1 & X4X1 & X4X1 & X4X1 & X4X1 & X4X1 \\
\hline & & M3M2 & X4X5X3 & X4X5X3 & X4X5X3 & X4X5X3 & X4X5X3 & X3X2X1 \\
\hline & & & M3X1 & X3M5X1 & X3X2X1 & X3X2X1 & X3X2X1 & X3X5 \\
\hline & & & M3M5 & X3M4M5 & X3X5X1 & X3X5X1 & X3X5X1 & \\
\hline & & & & & X3X2M5 & X3X2X3X5 & X2X3X5 & \\
\hline & & & & & X3X5M5 & X3X5X3x5 & X3X5 & \\
\hline
\end{tabular}

\section{Conclusion}

In the process of generating steam turbine unit, it is necessary to solve the problem of fault analysis and collection. In this process, can not be accurate enough and fault reason, logic reason of each fault has problems, it needs rich experience in the field, but a large number of researchers in the research process is often less practical, a vibration fault of steam turbine read without thorough understanding, in different units is not the same and even in the same unit in different period of time, 
the environment is different, this is an obstacle for constructing fault tree. But we are still in the actual process of fault tree can provide the basis for the vibration diagnosis, this method is not accurate enough, to be with the help of the computer, because the computer can deal with a large amount of information timely, fast to each actual occurrence probability and the present fault reason. In addition, the difficulty of quantitative analysis of turbine vibration fault tree lies in the need for a large number of accurate data, which is a big problem for the researchers in the laboratory. Finally, if the steam turbine vibration fault tree to draw out, is very large, so in qualitative analysis and quantitative analysis when the combinatorial explosion problem, and solve the problem now is not mature, most can only use the simplified means, myopia, error and mathematics to solve.

\section{References}

[1] L.P.Li.Fault diagnosis of steam turbine [M]. Beijing: China Electric Power Press, 2002

[2] B.Wu,Y.X.Ma.Expert system [M]. Beijing: Beijing Institute of Technology press, 2001.

[3] C.Zhang. Intelligent fault diagnosis of Steam Turbine Shafting Based on support vector machine [D].Baoding: North China Electric Power University, 2009.

[4] Sam Y S,Randall R B.Condition Monitoring of Electric Motors by Analysis of Current Waveform[J].IEEE.

[5] Adam Adgar,Chris Cox,John Macintyre. Automatic Fault Diagnosis for Machinery using Statistical and Neural Network Techniques[J].COMADEM Proceedings, 1998.

[6] Chevalier R,Brasseurs. PSAD: Condition Based in maintenance Through Permanent Monitoring[J].IFTOMM,UDINE 1998,12.

[7] Schiff T. The equipment Reliability Program at Willamette Industries' Kingsport, TN Plant[J]. Willamette Industries, 1998.

[8] Roemer M, Mauney D. Turbine-generator Maintenance Outage Optimization[J]. Predictive Maintenance Technology National Conference,1998,Nov.Indiana,USA.

[9] ISO 13379: 2003 Condition Monitoring and Diagnosis of Machines-General Guidelines on Data Interpretation and Diagnostics Techniques. 